\title{
Development of a Model for Electronic Toll-Collection System
}

\author{
Md. Farhad Ismail*, M.A.R. Sarkar \\ Department of Mechanical Engineering, Bangladesh University of Engineering \& Technology (BUET), Dhaka, \\ Bangladesh \\ *Email: farhadananda.128@gmail.com
}

\begin{abstract}
The paper presents a new method for electronic toll collection system and to match car number plate detection system. The electronic toll collection system (ETS) has been fabricated based on microcontroller. Here a system is developed to collect tolls according to the weight of the vehicle. The car number plate detection method utilizes template matching technique to approximate the location of car number plate. Then, using this output from the template matching method, color information is used to eliminate the unwanted color areas from the approximate number plate region without affecting the correct color regions. Hence, the number plate region can be determined more accurately. This work can easily be done by image processing system using MATLAB. The method has low complexity and reduced the processing time magnificently. This automated system also shows a better performance in highway traffic management. This paper shows the gateway to fabricate a highly automated toll-plaza.
\end{abstract}

Index Terms - Automated, Electronic toll system (ETS), MICROCONTROLLER, Traffic management, Image processing.

\section{INTRODUCTION}

The purpose of this paper is to collect toll according to the weight of the vehicles and to build a real time application which recognizes license plates from cars at a gate, for example at the entrance of a parking area. The system, based on regular PC with video camera, catches video frames which include a visible car license plate and processes them. Once a license plate is detected, its digits are recognized, displayed on the User Interface or checked against a database. The focus is on the design of algorithms used for extracting the license plate from a single image, isolating the characters of the plate and identifying the individual characters. Electronic Toll Collection (ETC) is a technology that allows for electronic payment of tolls. The endeavor of our work was to develop a highly automated toll-collection system. The automated

Manuscript received January 21, 2012; revised February 15, 2012; accepted February 20, 2012. system classifies [1] a vehicle based on its load range. In recent world, while collecting tolls in the bridges/ tunnels there may arise various problems such as: lengthy process for money transactions, which leads long traffic congestion as well. Here in Bangladesh the toll collection system is not developed. Moreover, it is time consuming and not accurate. The overloaded vehicles give the same amount of toll like the unloaded one though the overloaded vehicles damage the bridge more than that of the unloaded one. In some cases the vehicles carry a huge amount of load which crosses the legal limit. Moreover, corruption is also a major problem here. This is why; such a toll collection system is fabricated here. This toll-collection system is designed for achieving a fraud free and completely auditable operation by accurately detecting and registering vehicle movements and toll collector's activities. A central control system is introduced here for online monitoring of the tool-plaza. A pressure sensor is used to measure the weight of the vehicles which gives analogous data to the microcontroller. The microcontroller takes the data from the pressure sensor and gives the output data as a unit of currency. A system is also introduced here to control the barricade with the micro controller [2]. Whenever the load of the vehicle is measured then the toll is collected and then the barricade is opened. It just takes a few moments and by doing this a better traffic management is also confirmed as well as the accurate measurement of the load of the vehicle.

The objectives of image processing based electronic toll collection are many. It gives an accurate data as well as saves the license plate of the vehicles to recognize any unwanted vehicle.

\section{HARDWARE SETUP}

The hardware part of the system covers the whole electrical system. A model toll-plaza was designed [3] by wooden frame, which gives a pictorial view of the proposed automated toll-plaza (Fig. 1). 


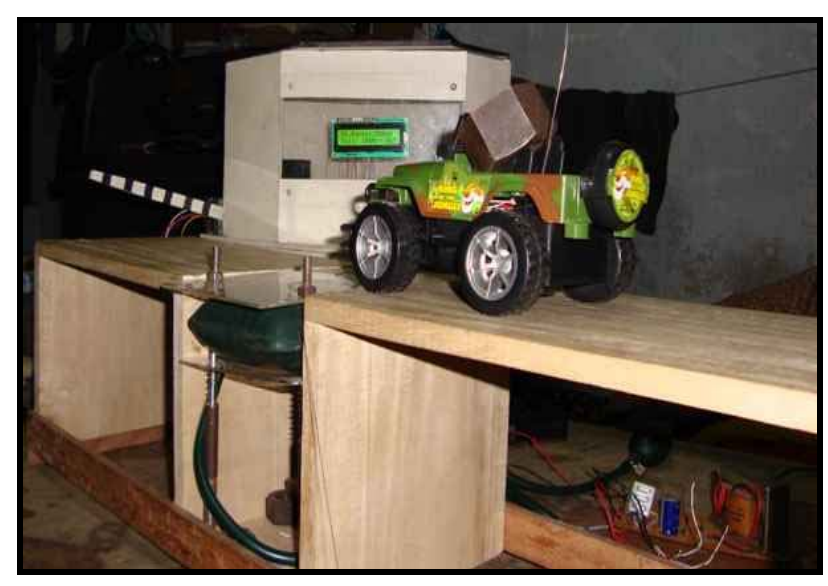

Fig. 1: Model of an automatic highway toll-plaza

\section{A. WEBCAM}

It has been used to capture the images of the number plates of the vehicles. The webcam (Fig. 2) is controlled by the pc USB port. When the car stays on the stage of the weight sensor the control unit then gives command to the computer to capture the image of the number plate.

\section{B. Barricade}

For proper traffic management, a barricade is used near the toll-plaza. It also ensures toll collection from every vehicle that passes thorough the toll booth. Here a stepper motor is used to control the barricade. The motor is fully automated by the micro controller. A stepper motor (or step motor) is a brushless, synchronous electric motor that can divide a full rotation into a large number of steps. Here a uni-poller stepper motor is used.

\section{C.LCD Display}

A LCD display is used here to give the output amount. A liquid crystal display (LCD) is a thin, flat panel used for electronically displaying [4] information such as text, images, and moving pictures. The LCD display is interfaced with the microcontroller.

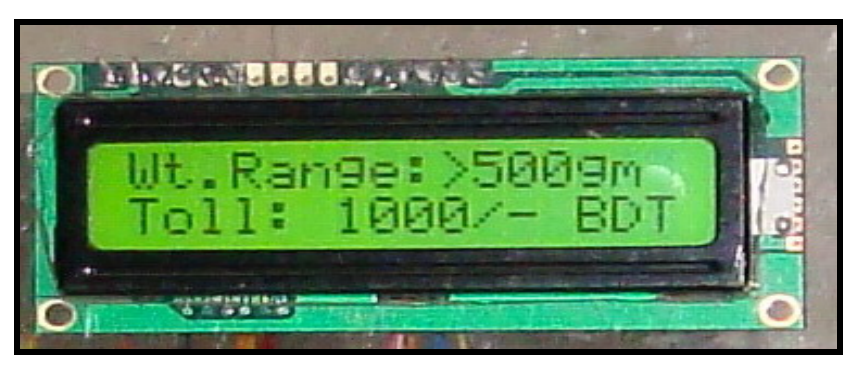

Fig. 2 WEBCAM used in the toll booth

\section{Coin Counter Machine}

A coin counter machine is also integrated with the circuit. Here a coin counter machine is developed. But card punching system can also be integrated in stead of coin machine. Whenever the required number of coin is inserted then the microcontroller gives the command to the stepper motor to open the barricade. When another vehicle comes on the weight sensing stage it then immediately gives command to close the barricade. Fig. 3 shows a brief review of the system.

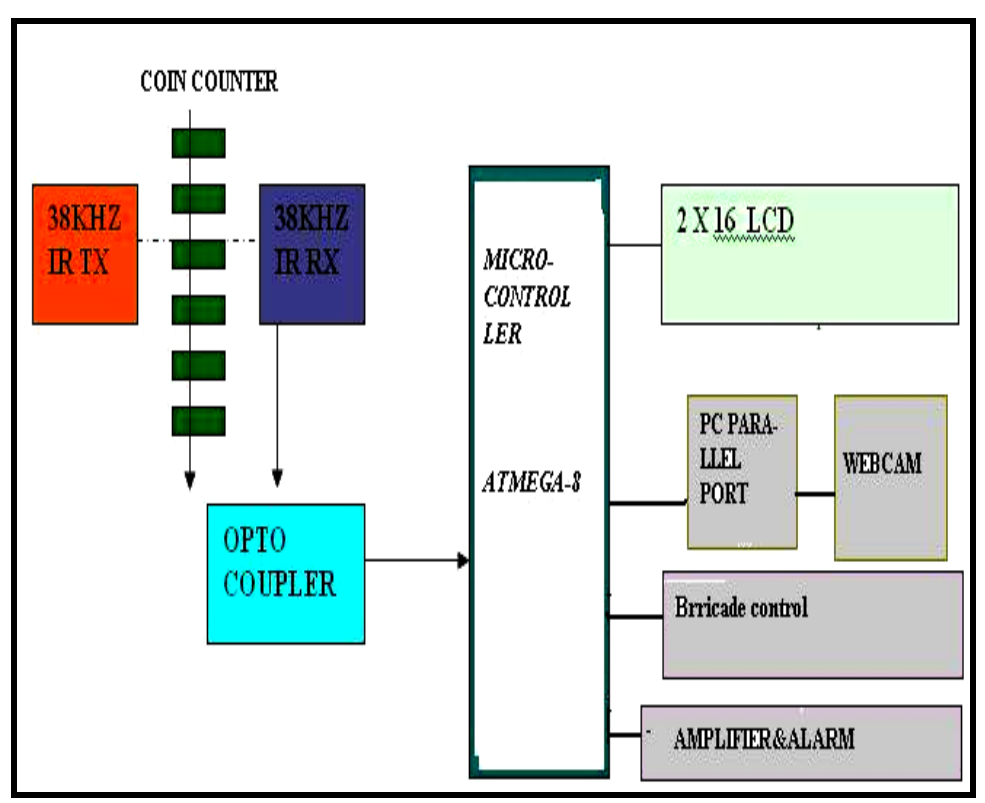

Fig. 3 Brief overview of the system

\section{E. Alarming system}

An alarming system is also integrated with the microcontroller. An amplifier and an alarming speaker are also attached with the microcontroller.

\section{SENSOR SYSTEM AND ACTUATORS}

Sensors are generally used to convert a physical signal into corresponding electrical signal. Then by means of proper amplification or attenuation, it is converted into numeric quantity. The sensors that are used can be listed as below:

A) MPXV 5050gp (pressure sensor).

B) Pressure bag.

\section{A. MPXV 5050gp}

One of the important part of our experiment is the pressure sensor. Here the pressure sensor MPXV 5050gp is used. MPX5050GP piezoresistive transducer is a monolithic silicon pressure sensor (Fig. 4) that combines advanced micromachining techniques, thin-film metallization, and bipolar processing to provide an accurate, high level analogue output signal that is proportional to the applied pressure. The MPX5050/MPXV5050G series pressure sensor [5] operating characteristics and internal reliability and qualification tests are based on use of dry air as the pressure media. 


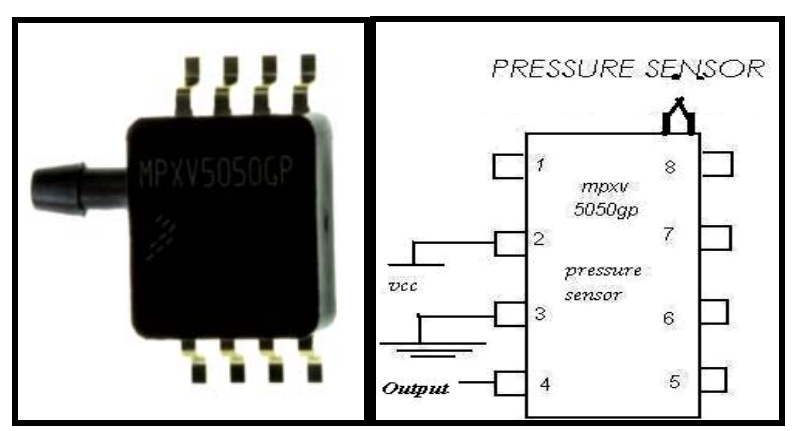

Fig. 4: Pressure sensor and circuit diagram

The output voltage of the pressure sensor is almost proportional to the pressure imposed upon it (Fig. 5). The MPX5050/MPXV5050G series pressure sensor operating characteristics and internal reliability and qualification tests are based on use of dry air as the pressure media. Media, other than dry air, may have adverse effects on sensor performance and long-term reliability.

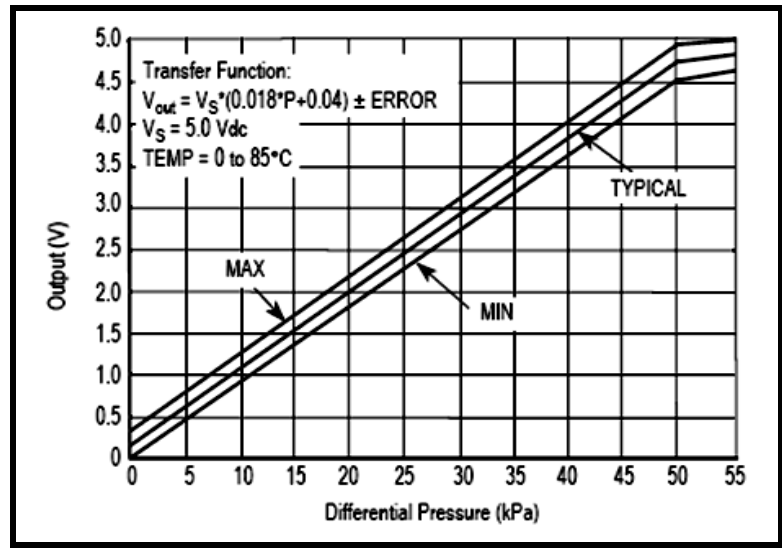

Fig. 5: Differential pressure vs. output curve for MPX5050

\section{A. Pressure Bag}

A pressure bag is used here under the stage where the loads of the vehicles are measured. Whenever a vehicle stays on the stage it then gives a pressure to the pressure bag. The pressure bag which is filled with dry air is then compressed and gives the input of the pressure sensor. The pressure sensor was calibrated according to weight (Fig. 6).

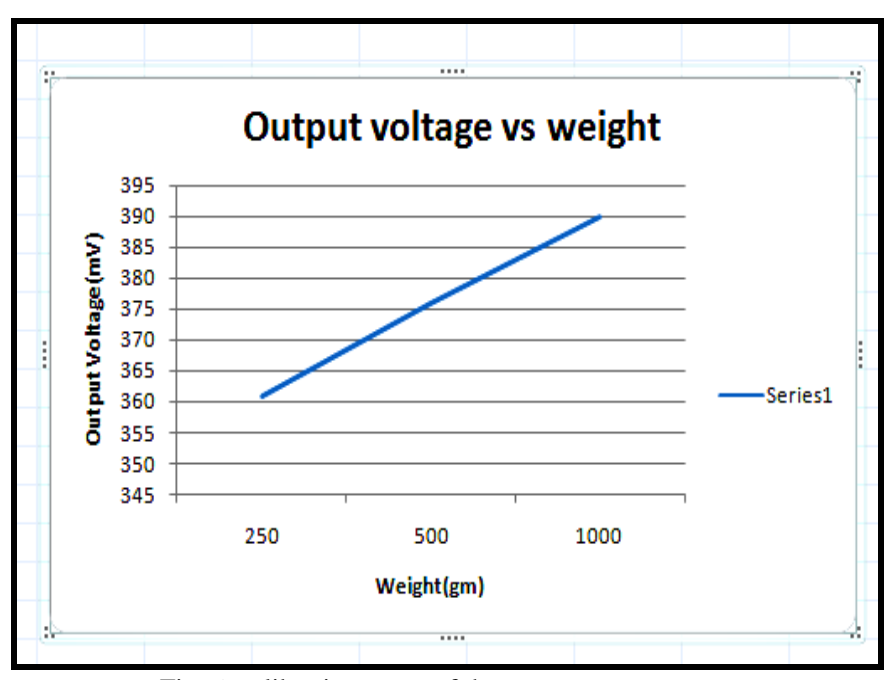

Fig. 6: calibration curve of the pressure sensor

\section{CONTROL SYSTEM}

A well developed control system is necessary to combine all the sensors and actuators. The control system is designed as an integrated intelligence system. The control system contains code, to perform all the tasks.

\section{A. Microcontroller Part}

Here microcontroller ATMEGA-8 is used. The microcontroller has 28 pins. Here the ADC0 pin is used to take the data from the pressure sensor. Then the microcontroller just converts the data into the amount of toll within a certain limit. The amount of toll as well as the weight range of the vehicle is then displayed through the LCD display. PortD of the microcontroller was set for the display (Fig. 7). PortC is also used to count the coins by the receiver and transmitter sensor. PortB is set to control the barricade and to give the input [6] to the PC to capture the image of the number plate of the vehicle.

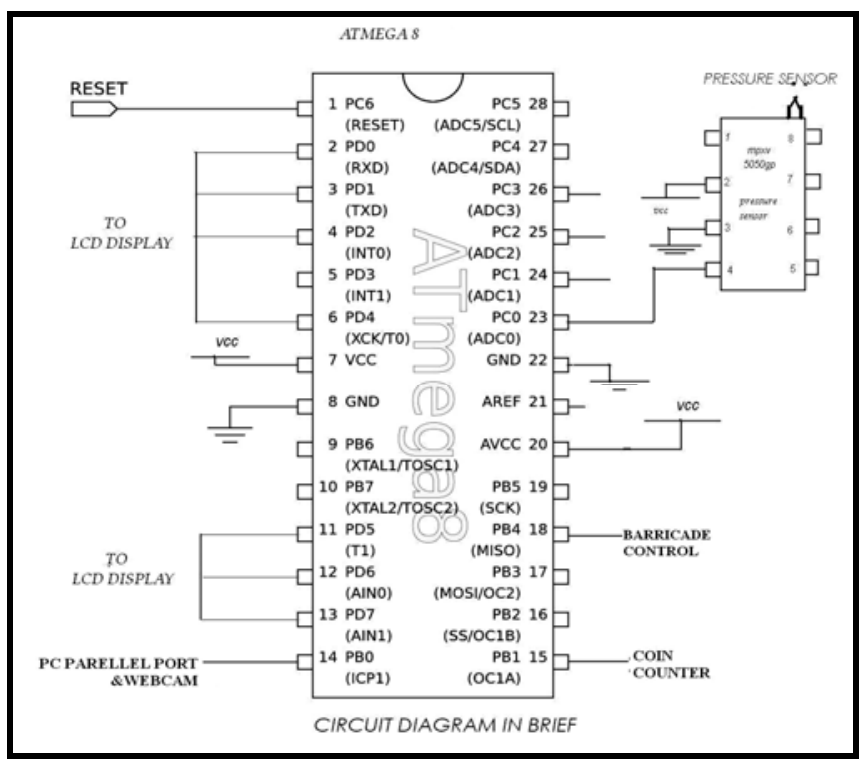

Fig. 7 pin configuration of ATMEGA 8 


\section{B. Barricade control system}

Here the barricade is controlled by the uni-polar stepper motor (Fig. 8). The stepper motor is run by the ULN2003 IC. This IC is coupled with the PortB of the microcontroller.

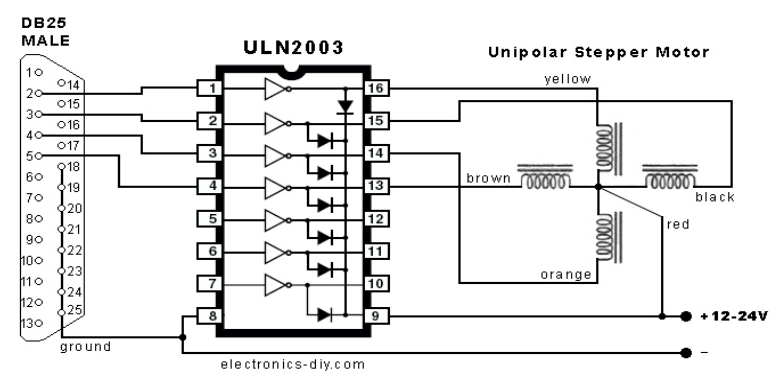

Fig. 8 Stepper-motor circuit diagrams

\section{IMAGE PROCESSING PART}

It is a very important part to ensure the security system. Every vehicle should be monitored by the close circuit cameras and the webcams. A digital image is composed of pixels which can be thought of as small dots on the screen. The word pixel comes from "picture element". The resolution of an image is described as the number of pixels horizontally times the number of pixels vertically. A digital image is an instruction of how to color each pixel. A typical size of an image is 512-by-512 pixels. For example: $2^{9}=512$. In the general case an image is of size m-by-n if it is composed of $m$ pixels in the vertical direction and $n$ pixels in the horizontal direction. Let an image is on the format 512-by-1024 pixels. This means that the data for the image must contain information about 524288 pixels, which requires a lot of memory! Hence, compressing images is essential for efficient image processing. Fourier analysis and Wavelet analysis can help to compress an image significantly. There are also a few "computer scientific" tricks (for example entropy coding) to reduce the amount of data required to store an image. We will refer to a pixel by the number of its row and the number of its column. By this convention, the $\mathrm{x}$-axis is vertical and the $y$-axis is horizontal. This is consistent with the way we refer to the elements of a matrix (Fig. 9).

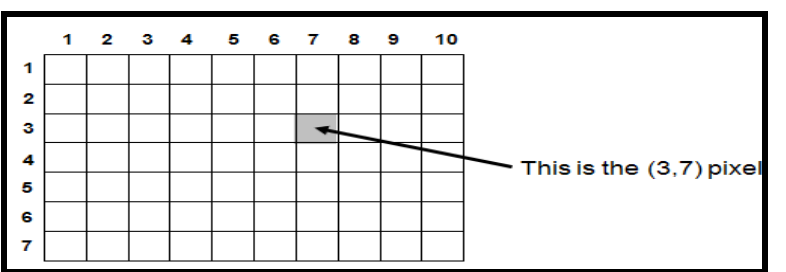

Fig. 9. A 10x7 image

Most images are JPEG-images which is the name for one of the most widely used compression standards for images. For example, an image named myimage.jpg is stored in the JPEG format and we will see on that we can load an image of this format into MATLAB. If an image is stored as a JPEG-image on the disc we first read it into MATLAB. However, in order to start working with an image, for example perform a wavelet transform on the image, it must be converted into a different format. This section explains four common formats. There are few methods that can be used for character recognition, namely.

\section{A. Neural network based method}

A neural network is a mathematical model for information processing based on a connectionist approach to computation. The way of connection is depends on the particular model. For character recognition, 2 type of algorithm can be use, that is Back Propagation and constraint based decomposition (CBD). In both methods, training is required to increase the accuracy of recognition.

\section{B. Template matching}

The templates of all the character will be define with some test point. The character which wishes to be recognized will be compare with those templates. The template with the highest matched point will be the character appears in that image. This paper will discuss on the software development for an access control application base on the LPR technology. The system is capable to recognizing car plate number automatically. After recognition, the plate number will be compared with the list of plate numbers in a database. If the number is inside the list, then the system will allow the particular car to enter the facility. This system consists of both hardware and software.

The process of image processing more or less consists of five steps. They are described below-

\section{Image representation}

1. Grayscale: A grayscale image (Fig. 10) $M$ pixels tall and $N$ pixels wide is represented as a matrix of double data type of size $M \times N$. Element values (e.g., My Image $(m, n)$ ) denote the pixel grayscale intensities in $[0,1]$ with $0=$ black and $1=$ white.

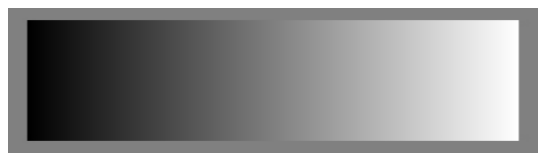

Fig. 10: Gray scale image

2. True color RGB: A true color red-green-blue (RGB) image (Fig. 11) is represented as a three-dimensional $M \times N \times 3$ double matrix [7]. Each pixel has red, green, blue components along the third dimension with values in [0,1], for example, the color components of pixel $(m, n)$ are $\mathrm{My}$ Image $(m, n, 1)=$ red, My Image $(m, n, 2)=$ green, My Image $(m, n, 3)=$ blue. 


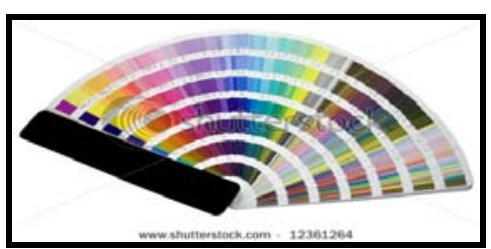

Fig. 11: True color RGB image

3. Indexed: Indexed (palette) images are represented with an index matrix of size $M \times N$ and a color map matrix of size $K \times 3$. The color map holds all colors used in the image and the index matrix represents the pixels by referring to colors in the color map. For example, if the 22nd color is magenta $\operatorname{MyColormap}(22,:)=[1,0,1]$, then $\operatorname{My} \operatorname{Image}(m, n)=$ 22 is a magenta-colored pixel.

4. Binary: A binary image (Fig. 12) is represented by an $M \times N$ logical matrix where pixel values are 1 (true) or 0 (false).

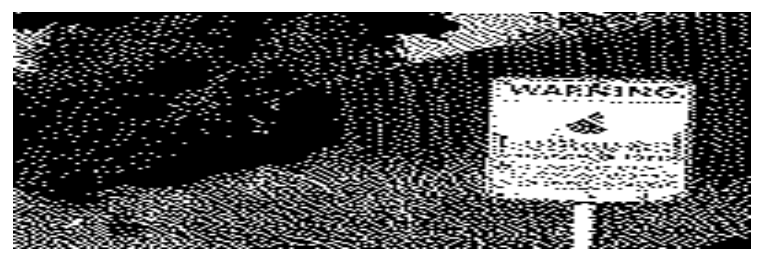

Fig. 12: Binary image

A binary image is a digital image that has only two possible values for each pixel. Typically the two colors used for a binary image are black and white though any two colors can be used. The color used for the object(s) in the image is the foreground color while the rest of the image is the background color. Binary images are also called bi-level or two-level. This means that each pixel is stored as a single bit ( 0 or 1$)$. So in binary image 1 bit is used for each pixel. A binary image is usually stored in memory as a bitmap, a packed array of bits. A $640 \times 480$ image requires a total of 307200 pixels that indicates a total of 307200 bits or 38400 bytes.

5. uint8: This type uses less memory and some operations compute faster than with double types. For simplicity, this tutorial does not discuss uint8 further.

\section{A. Reading and writing image files}

MATLAB can read and write images with the imread (read image) and imwrite (write image) built in function [8]. Although a fair number of file formats are supported, some are not. When reading images, an unfortunate problem is that imread returns the image data in uint8 data type, which must be converted to double and rescaled before use. So instead of calling imread directly, use the following M-file function to read and convert images

\section{B. Linear filters}

Linear filtering (Fig. 13) is the cornerstone technique of signal processing. To briefly introduce, a linear filter is an operation where at every pixel $x_{m, n}$ of an image, a linear function is evaluated on the pixel and its neighbors to compute a new pixel value $y_{m, n}$.

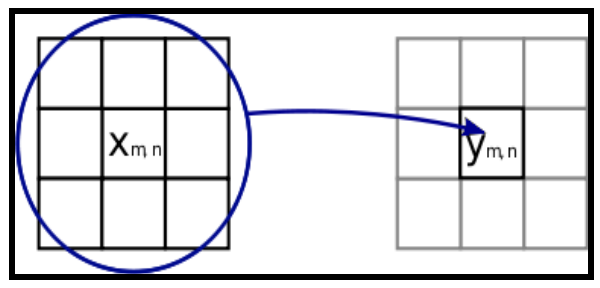

Fig. 13: A linear filter in two dimensions has the general form

$$
y_{m, n}=\sum_{j} \sum_{k} h_{j, k} x_{m-j, n-k}
$$

Where $x$ is the input, $y$ is the output, and $h$ is the filter impulse response. Different choices of $h$ lead to filters that smooth, sharpen, and detect edges, to name a few applications. The right-hand side of the above equation is denoted concisely as $h * x$ and is called the "convolution of $h$ and $x$.”

\section{Nonlinear filters}

A nonlinear filter is an operation where each filtered pixel $y_{m, n}$ is a nonlinear function of $x_{m, n}$ and its neighbors. Here we briefly discuss a few types of nonlinear filters.

Grayscale is usually the preferred format for image processing. In cases requiring color, an RGB color image can be decomposed and handled as three separate grayscale images. Indexed images must be converted to grayscale or RGB for most operations. In photography and computing, a grayscale or grayscale digital image is an image in which the value of each pixel is a single sample, that is, it carries only intensity information. Images of this sort, also known as black-and-white, are composed exclusively of shades of gray, varying from black at the weakest intensity to white at the strongest. Grayscale images are distinct from one-bit black-and-white images, which in the context of computer imaging are images with only the two colors, black, and white. Grayscale images have many shades of gray in between. The intensity of a pixel is expressed within a given range between a minimum and a maximum, inclusive. This range is represented in an abstract way as a range from 0 (total absence, black) and 1 (total presence, white), with any fractional values in between. The high values correspond to bright pixels and the low values correspond to dark pixels. The whole image is described by an array of numbers called matrix.

\section{License Plate Matching}

Template matching (Fig. 14) or license plate matching [9] is a technique compares portions of images against one another. The software design and development are involving the following things: A Graphical User Interface 
(GUI) (Fig. 14) application window as the main control panel of the system. Image processing module that analyzes the image of a car and return the plate number. The tools used for software development is Microsoft Visual Basic 6.0 and MATLAB 7.0. The image processing module is developed using MATLAB while the other module is built using Visual Basic (VB). The image processing module will then compiled into a dynamic linkage library (dll). Sample of image may be used to recognize similar objects in source image. If standard deviation of the template image compared to source image is small enough, template matching [10-12] may be used. Templates are most often used to identify printed characters, small object, and car number plate.

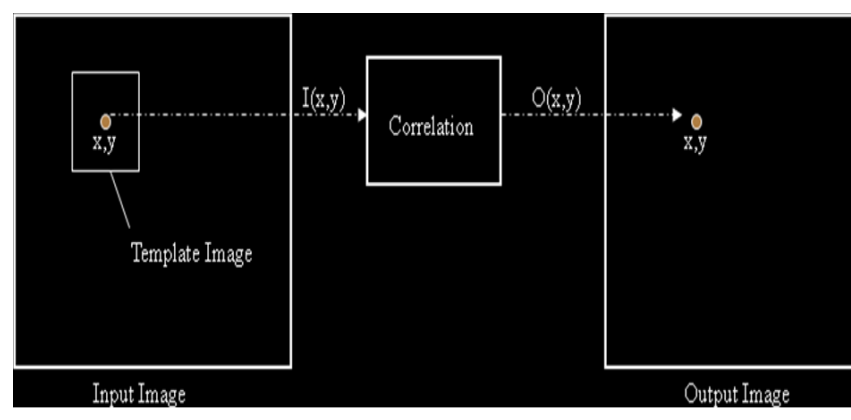

Fig. 14: Template matching process

The matching process moves the template image to all possible positions in a larger source image and computes a numerical index that indicates how well the template matches the image in that position. Match is done on a pixel by pixel basis. In order to have rough detection of the number plate region, template matching technique is used. The template size is $166 \times 59$ pixels and has a random white box pattern that has the element of the number plate region to minimize the false detection. In this paper, template size already been fixed to reduce the processing time. This leads to inaccurate detection.

To correct the uneven illumination of the input image, top hat filtering must be applied in order to get the lowest white region without affecting the number plate clarity. Mask of disk shape structuring element with 12 pixels distance from the structuring origin to the point of the disk must be applied to the input image.

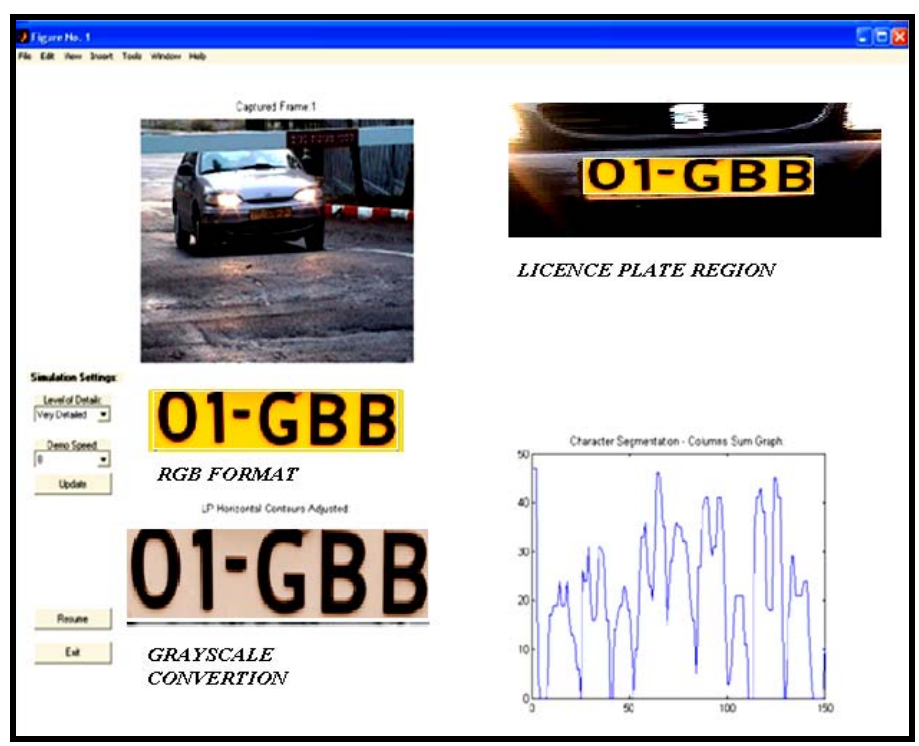

Fig. 15: GUI of license matching using MATLAB

\section{SUMMARY}

The designed toll-collection system reduces the collection time and corruption and thus increases the government revenue. The toll-collection system also ensures safety and better traffic management.

\section{ACKNOWLEDGMENT}

This work was supported by control \& Instrumentation lab of Bangladesh University of Engineering \& Technology (BUET).

\section{REFERENCES}

[1] Celil Ozkurt and Fatih Camc, "Automatic Traffic Density Estimation and Vehicle Classification for Traffic Surveillence System Using Neural Networking”, Mathematical and Computational Applications, Vol. 14, No. 3, pp. 187-196, 2009.

[2] J. Nehrbass, S. Samsi, J. Carlos Chaves, J.Unpingco, B. Guilfoos, S. Ahalt, "Interfacing PC -based MATLAB Directly to HPC Resources”, published as graduate thesis, Ohio Supercomputer center, Colombus, OH. 2006.

[3] Teruaki Ito and Tomoyuki Hiramoto, “ Towards Collaboration Communication Using Simulation-Based Traffic Model”, published as funded research, Department of Mechanical Engineering, University of Tokushima, Japan

[4] X. Quintana1, P.L. Castillo1, J.M. otón*1, N. Bennis1, A. Lara1, V. Urruchi2, and R. D¥browski3, "Video-rate multiplexed driving scheme for passive antiferroelectric liquid cryatal display”, 15 th Liquid Crystal Conference, Warsaw Poland, 2004. Pp:291-297.

[5] www.alldatasheet.com/MPXV5050 gp

[6] A. Oh, "The CMS DAQ and Run Control System”, Journal of Physics, Conference series 1102008 (092020).

[7] Wu Wei, Mingjun Wang, Zhongxiang Huang, "An automatic method of location for number plate using color features, " in International Conference on Image Processing, Thessaloniki, Greece, 2001, pp. 782-785.

[8] http://www.accessmylibrary.com 
[9] Jun-Wei Hsieh, Shih-Hao Yu, and Yung-Sheng Chen, "Morphology-based license plate detection from complex scene, " in Proceedings of 16th International Conference on Pattern Recognition, vol3, 2002, pp. 176-179.

[10] A. S. Johnson, B. M. Bird, "Number plate matching for automatic vehicle identification," IEE Colloquium on Electronic Image and Image Processing in Security and Forensic, 1990.

[11] A.A. Rokoni, M.F. Ismail, M.O. Reza, M.A.R. Sarkar, "Development of an Image Processing Based Container Traffic Control System”, International Conference on Mechanical, Automotive and Aerospace Engineering-ICMAAE-2011, Paper ID-13, Kuala Lumpur, Malaysia, 17-19 May, 2011.

[12] M.F. Ismail, “Automatic Highway Tool Collection”, the project was carried out in ME-361 course, January 2010, Mechanical Engineering Department, BUET.

Md. Farhad Ismail, male, BUET, Dhaka, Research Engineer, B.Sc., his research interest includes Automation, Mechatronics and embedded control systems. His research interests also include thermo-fluid dynamics and CFD simulation.

M. A. Rashid Sarkar, male, BUET, Dhaka, Professor, Ph.D, his research directions include Instrumentation, optimal control and thermal engineering. 